\title{
Moufang transformations and Noether currents
}

\author{
Eugen Paal \\ Department of Mathematics \\ Tallinn University of Technology \\ Ehitajate tee 5, 19086 Tallinn, Estonia \\ E-mail: eugen.paal@ttu.ee
}

\begin{abstract}
The Noether currents generated by continuous Moufang tranformations are constructed and their equal-time commutators are found. The corresponding charge algebra turns out to be a birepresentation of the tangent Mal'ltsev algebra of an analytic Moufang loop.
\end{abstract}

\section{Introduction}

The Noether currents generated by the Lie transformation groups are well known and widely exploited in modern field theory and theory of elementary particles. Nevertheless, it may happen that group theoretical formalism of physics is too rigid and one has to extend it beyond the Lie groups and algebras. From this point of view it is interesting to elaborate an extension of the group theoretical methods based on Moufang loops as a minimal nonassociative generalization of the group concept. In particular, the Mal'tsev algebra structure of the quantum chiral gauge theory was established in [1, 2.

In this paper, the Noether currents generated by continuous Moufang tranformations (Sec. 2) are constructed. The method is based on the generalized Lie-Cartan theorem (Sec. 3). It turns out that the resulting charge algebra is a birepresentation of the tangent Mal'ltsev algebra of an analytic Moufang loop (Sec. 4). Throughout the paper $\mathrm{i} \doteq \sqrt{-1}$. 


\section{Moufang loops and Mal'tsev algebras}

A Moufang loop [3, 4] is a quasigroup $G$ with the unit element $e \in G$ and the Moufang identity

$$
(a g)(h a)=a(g h) a, \quad a, g, h \in G .
$$

Here the multiplication is denoted by juxtaposition. In general, the multiplication need not be associative: $g h \cdot a \neq g \cdot h a$. Inverse element $g^{-1}$ of $g$ is defined by

$$
g g^{-1}=g^{-1} g=e .
$$

A Moufang loop $G$ is said [5] to be analytic if $G$ is also a real analytic manifold and main operations - multiplication and inversion map $g \mapsto g^{-1}$ - are analytic mappings.

As in the case of the Lie groups, structure constants $c_{j k}^{i}$ of an analytic Moufang loop are defined by

$$
\left.c_{j k}^{i} \doteq \frac{\partial^{2}\left(g h g^{-1} h^{-1}\right)^{i}}{\partial g^{j} \partial h^{k}}\right|_{g=h=e}=-c_{k j}^{i}, \quad i, j, k=1, \ldots, r \doteq \operatorname{dim} G .
$$

Let $T_{e}(G)$ be the tangent space of $G$ at the unit element $e \in G$. For any $x, y \in T_{e}(G)$, their (tangent) product $[x, y] \in T_{e}(G)$ is defined in component form by

$$
[x, y]^{i} \doteq c_{j k}^{i} x^{j} y^{k}=-[y, x]^{i}, \quad i=1, \ldots, r .
$$

The tangent space $T_{e}(G)$ being equipped with such an anti-commutative multiplication is called the tangent algebra of the analytic Moufang loop $G$. We shall use notation $\Gamma \doteq\left\{T_{e}(G),[\cdot, \cdot]\right\}$.

The tangent algebra of $G$ need not be a Lie algebra. There may exist such a triple $x, y, z \in T_{e}(G)$ that does not satisfy the Jacobi identity:

$$
J(x, y, z) \doteq[x,[y, z]]+[y,[z, x]]+[z,[x, y]] \neq 0 .
$$

Instead, for any $x, y, z \in T_{e}(G)$ one has a more general Mal'tsev identity [5]

$$
[J(x, y, z), x]=J(x, y,[x, z]) .
$$

Anti-commutative algebras with this identity are called the Mal'tsev algebras. Thus every Lie algebra is a Mal'tsev algebra as well. 


\section{Birepresentations}

Consider a pair $(S, T)$ of the maps $g \mapsto S_{g}, g \mapsto T_{g}$ of a Moufang loop $G$ into $G L_{n}$. The pair $(S, T)$ is called a (linear) birepresentation of $G$ if the following conditions hold:

- $S_{e}=T_{e}=\mathrm{id}$,

- $T_{g} S_{g} S_{h}=S_{g h} T_{g}$,

- $S_{g} T_{g} T_{h}=T_{h g} S_{g}$.

The birepresentation $(S, T)$ is called associative, if the following simultaneous relations hold:

$$
S_{g} S_{h}=S_{g h}, \quad T_{g} T_{h}=T_{h g}, \quad S_{g} T_{h}=T_{h} S_{g} \quad \forall g, h \in G
$$

In general, one can consider nonassociative birepresentations even for groups.

The generators of a differentiable birepresentation $(S, T)$ of an analytic Moufang loop $G$ are defined as follows:

$$
\left.S_{j} \doteq \frac{\partial S_{g}}{\partial g^{j}}\right|_{g=e},\left.\quad T_{j} \doteq \frac{\partial T_{g}}{\partial g^{j}}\right|_{g=e}, \quad j=1, \ldots, r .
$$

Generalized Lie-Cartan Theorem $1([6,6])$. The generators of a differentiable birepresentation of an analytic Moufang loop satisfy the commutation relations

$$
\begin{aligned}
& {\left[S_{j}, S_{k}\right]=2 Y_{j k}+\frac{1}{3} c_{j k}^{p} S_{p}+\frac{2}{3} c_{j k}^{p} T_{p}} \\
& {\left[S_{j}, T_{k}\right]=-Y_{j k}+\frac{1}{3} c_{j k}^{p} S_{p}-\frac{1}{3} c_{j k}^{p} T_{p}} \\
& {\left[T_{j}, T_{k}\right]=2 Y_{j k}-\frac{2}{3} c_{j k}^{p} S_{p}-\frac{1}{3} c_{j k}^{p} T_{p}}
\end{aligned}
$$

where the Yamaguti operators $Y_{j k}$ obey the relations

$$
\begin{gathered}
Y_{j k}+Y_{k j}=0 \\
c_{j k}^{p} Y_{p l}+c_{j k}^{p} Y_{p l}+c_{j k}^{p} Y_{p l}=0 .
\end{gathered}
$$

and satisfy the reductivity relations

$$
\begin{aligned}
& {\left[Y_{j k}, S_{n}\right]=d_{j k n}^{p} S_{p}} \\
& {\left[Y_{j k}, T_{n}\right]=d_{j k n}^{p} T_{p}}
\end{aligned}
$$


and commutation relations

$$
\left[Y_{j k}, Y_{l n}\right]=d_{j k l}^{p} Y_{p n}+d_{j k n}^{p} Y_{l p}
$$

with the Yamaguti constants

$$
6 d_{j k l}^{p} \doteq c_{j s}^{p} c_{k l}^{s}-c_{k s}^{p} c_{j l}^{s}+c_{s l}^{p} c_{j k}^{s}
$$

Dimension of this Lie algebra does not exceed $2 r+r(r-1) / 2$. The Jacobi identities are guaranteed by the defining identities of the Lie [8] and general Lie 9] triple systems associated with the tangent Mal'tsev algebra $T_{e}(G)$ of $G$.

These commutation relations are known from the theory of alternative algebras [10]. This is due to the fact that commutator algebras of alternative algebras are Mal'tsev algebras. In a sense, one can also say that the differential of a birepresentation $(S, T)$ of the analytic Moufang loop is a birepresentation of its tangent Mal'tsev algebra $\Gamma$.

\section{Moufang-Noether currents and ETC}

Let us now introduce conventional canonical notations. The coordinates of a space-time point $x$ are denoted by $x^{\alpha}(\alpha=0,1, \ldots, d-1)$, where $x^{0}=t$ is the time coordinate and $x^{i}$ are the spatial coordinates denoted concisely as $\mathbf{x} \doteq\left(x^{1}, \ldots, x^{d-1}\right)$. The Lagrange density $\mathrm{E}[u, \partial u]$ is supposed to depend on a system of independent (bosonic or fermionic) fields $u^{A}(x)(A=1, \ldots, n)$ and their derivatives $\partial_{\alpha} u^{A} \doteq u_{\alpha}^{A}$. The canonical $d$-momenta are denoted by

$$
p_{A}^{\alpha} \doteq \frac{\partial \mathrm{E}}{\partial u_{\alpha}^{A}}
$$

The Moufang-Noether currents are defined in vector (matrix) notations as follows:

$$
s_{j}^{\alpha} \doteq p^{\alpha} S_{j} u, \quad t_{j}^{\alpha} \doteq p^{\alpha} T_{j} u
$$

and the corresponding Moufang-Noether charges are defined as spatial integrals by

$$
\sigma_{j}(t) \doteq-\mathrm{i} \int s_{j}^{0}(x) d \mathbf{x}, \quad \tau_{j}(t) \doteq-\mathrm{i} \int t_{j}^{0}(x) d \mathbf{x}
$$

By following canonical prescription assume that the following equal-time commutators (or anti-commutators when $u^{A}$ are fermionic fields) hold:

$$
\begin{aligned}
& {\left[p_{A}^{0}(\mathbf{x}, t), u^{B}(\mathbf{y}, t)\right]=-\mathrm{i} \delta_{A}^{B} \delta(\mathbf{x}-\mathbf{y})} \\
& {\left[u^{A}(\mathbf{x}, t), u^{B}(\mathbf{y}, t)\right]=0} \\
& {\left[p_{A}^{0}(\mathbf{x}, t), p_{B}^{0}(\mathbf{x}, t)\right]=0}
\end{aligned}
$$


As a matter of fact, these equal-time commutators (ETC) do not depend on the associativity property of either $G$ nor $(S, T)$. Nonassociativity hides itself in the structure constants of $G$ and in the commutators $\left[S_{j}, T_{k}\right]$. Due to this, the computation of the ETC of the Noether-Moufang charge densities can be carried out in standard way and nonassociativity reveals itself only in the final step when the commutators $\left[S_{j}, T_{k}\right]$ are required.

First recall that in associative case the Noether charge densities obey the ETC

$$
\begin{aligned}
{\left[s_{j}^{0}(\mathbf{x}, t), s_{k}^{0}(\mathbf{y}, t)\right] } & =\quad \mathrm{i} c_{j k}^{p} s_{p}^{0}(\mathbf{x}, t) \delta(\mathbf{x}-\mathbf{y}) \\
{\left[t_{j}^{0}(\mathbf{x}, t), t_{k}^{0}(\mathbf{y}, t)\right] } & =-\mathrm{i} c_{j k}^{p} t_{p}^{0}(\mathbf{x}, t) \delta(\mathbf{x}-\mathbf{y}) \\
{\left[s_{j}^{0}(\mathbf{x}, t), t_{k}^{0}(\mathbf{y}, t)\right] } & =0
\end{aligned}
$$

It turns out that for non-associative Moufang transformations these ETC are violated minimally. The Moufang-Noether charge density algebra reads

$$
\begin{aligned}
{\left[s_{j}^{0}(\mathbf{x}, t), s_{k}^{0}(\mathbf{y}, t)\right] } & =\quad \mathrm{i} c_{j k}^{p} s_{p}^{0}(\mathbf{x}, t) \delta(\mathbf{x}-\mathbf{y})-2\left[s_{j}^{0}(\mathbf{x}, t), t_{k}^{0}(\mathbf{y}, t)\right] \\
{\left[t_{j}^{0}(\mathbf{x}, t), t_{k}^{0}(\mathbf{y}, t)\right] } & =-\mathrm{i} c_{j k}^{p} t_{p}^{0}(\mathbf{x}, t) \delta(\mathbf{x}-\mathbf{y})-2\left[s_{j}^{0}(\mathbf{x}, t), t_{k}^{0}(\mathbf{y}, t)\right]
\end{aligned}
$$

The ETCs

$$
\left[s_{j}^{0}(\mathbf{x}, t), t_{k}^{0}(\mathbf{y}, t)\right]=\left[t_{j}^{0}(\mathbf{y}, t), s_{k}^{0}(\mathbf{x}, t)\right]
$$

represent associator of an analytic Moufang loop and so may be called the associator as well. Associator of a Moufang loop is not arbitrary but have to fulfil certain constraints [6], the generalized Lie and Maurer-Cartan equations. In the present sitiation the constraints can conveniently be listed by closing the above ETC, which in fact means construction of a finite dimensional Lie algebra generated by the Moufang-Noether charge densities.

Start by rewriting the Moufang-Noether algebra as follows:

$$
\begin{aligned}
& {\left[s_{j}^{0}(\mathbf{x}, t), s_{k}^{0}(\mathbf{y}, t)\right]=\mathrm{i}\left[2 Y_{j k}^{0}(x)+\frac{1}{3} c_{j k}^{p} s_{p}^{0}(x)+\frac{2}{3} c_{j k}^{p} t_{p}^{0}(x)\right] \delta(\mathbf{x}-\mathbf{y})} \\
& {\left[s_{j}^{0}(\mathbf{x}, t), t_{k}^{0}(\mathbf{y}, t)\right]=\mathrm{i}\left[-Y_{j k}^{0}(x)+\frac{1}{3} c_{j k}^{p} s_{p}^{0}(x)-\frac{1}{3} c_{j k}^{p} t_{p}^{0}(x)\right] \delta(\mathbf{x}-\mathbf{y})} \\
& {\left[t_{j}^{0}(\mathbf{x}, t), s_{k}^{0}(\mathbf{y}, t)\right]=\mathrm{i}\left[2 Y_{j k}^{0}(x)-\frac{2}{3} c_{j k}^{p} s_{p}^{0}(x)-\frac{1}{3} c_{j k}^{p} t_{p}^{0}(x)\right] \delta(\mathbf{x}-\mathbf{y})}
\end{aligned}
$$

Here (2) can be seen as a definition of the Yamagutian $Y_{j k}^{0}(x)$. The Yamagutian is thus a recapitulation of the associator. It can be shown that

$$
\begin{gathered}
Y_{j k}^{0}(x)+Y_{k j}^{0}(x)=0 \\
c_{j k}^{p} Y_{p l}^{0}(x)+c_{k l}^{p} Y_{p j}^{0}(x)+c_{l j}^{p} Y_{p k}^{0}(x)=0
\end{gathered}
$$


The constraints (4) trivially descend from the anti-commutativity of the commutator bracketing, but the proof of (5) needs certain effort. Further, it turns out that the following reductivity conditions hold:

$$
\begin{aligned}
& {\left[Y_{j k}^{0}(\mathbf{x}, t), s_{n}^{0}(\mathbf{y}, t)\right]=\mathrm{i} d_{j k n}^{p} s_{p}^{0}(\mathbf{x}, t) \delta(\mathbf{x}-\mathbf{y})} \\
& {\left[Y_{j k}^{0}(\mathbf{x}, t), s_{n}^{0}(\mathbf{y}, t)\right]=\mathrm{i} d_{j k n}^{p} s_{p}^{0}(\mathbf{x}, t) \delta(\mathbf{x}-\mathbf{y})}
\end{aligned}
$$

Finally, by using the redictivity conditions, one can check that the Yamagutian obeys the Lie algebra

$$
\left[Y_{j k}^{0}(\mathbf{x}, t), Y_{l n}^{0}(\mathbf{y}, t)\right]=\mathrm{i}\left[d_{j k l}^{p} Y_{p n}^{0}(\mathbf{x}, t)+d_{j k n}^{p} Y_{l p}^{0}(\mathbf{x}, t)\right] \delta(\mathbf{x}-\mathbf{y})
$$

When integrating the above ETC (1) - (8) one can finally obtain the

Theorem 2 (Moufang-Noether charge algebra). The Moufang-Noether charge algebra $(\sigma, \tau)$ is a birepresentation of the Mal'tsev algebra $\Gamma$.

The paper was in part supported by the Estonian Science Foundation, Grant 5634 .

\section{References}

[1] S.-G. Jo. Commutator in anomalous non-abelian chiral gauge theory. Phys Lett. B, 1985, 163, 353-359.

[2] A. J. Niemi and G. W. Semenoff. Quantum holonomy and the chiral gauge anomaly Phys. Rev. Lett. B, 1985, 55, 927-930.

[3] R. Moufang. Zur Struktur von Alternativkörpern. Math. Ann. B110 (1935), 416.

[4] H. Pflugfelder. Quasigroups and Loops: Introduction. Heldermann Verlag, Berlin, 1990.

[5] A. I. Mal'tsev. Analytical loops. Matem. Sbornik. 36 (1955), 569.

[6] E. Paal. Continuous Moufang transformations. Acta Appl. Math. 1998, Vol. 50, 77-91.

[7] E. Paal. Moufang loops and integrability of generalized Lie equations. Czech. J. Phys. Vol. 54 (2004), 1375-1380.

[8] O. Loos. Über eine Beziehung zwischen Malcev-Algebren und LieTripelsystemen. Pacific J. Math. 18 (1966), 553.

[9] K. Yamaguti. Note on Malcev algebras. Kumamoto J. Sci. A5 (1962), 203; A6 (1963), 9.

[10] R. D. Schafer. An Introduction to Nonassociative algebras. Academic Press, New York, 1966. 